\title{
DETERMINATION OF DETONABLE GAS MIXTURE HEAT FLUXES AT THERMAL DEBURRING
}

\author{
Sergey Plankovskyy ${ }^{a}$, Andrzei TeodorczyK $^{b}$, Olga Shypul $^{a}$, \\ Oleg Tryfonov ${ }^{a}$, Dmytro BregA ${ }^{a, *}$ \\ ${ }^{a}$ National Aerospace University "Kharkiv aviation institute", Aircraft Manufacturing Department, Kharkiv, \\ Ukraine \\ ${ }^{b}$ Warsaw University of Technology, Institute of Heat Engineering, Warsaw, Poland \\ * corresponding author: brega10.04@gmail.com
}

\begin{abstract}
Modern technology requires a multitude of precise parts that are a necessity in reliable methods of surface finishing. Energy of detonable gas mixture combustion has been used in manufacturing as a processing source for a long time. One of the most underappreciated methods is thermal deburring; this is caused by certain difficulties in modelling and simulation of this process due to a complex and poorly predictable nature of the combustion. A theoretical approach towards thermal deburring process using the conception of an equivalent chamber is described. Processing of combined experimental and computational data results in a simplified model of thermal deburring in the case with deflagration and combustion with a shock waves formation. The proposed mathematical model was verified by an experimental investigation of the combustion in a shock tube, the difference of compared parameters did not exceed $3 \%$. The heat fluxes at thermal deburring by combustible gas mixtures and their distribution on part surfaces according to the direction of the shock waves propagation were calculated. A relation between the value of the heat flux and shock waves propagation was found with a convincing repeating trend.
\end{abstract}

KEYWORDS: Thermal deburring, edge finishing, heat flux, shock waves, detonable gas mixtures.

\section{INTRODUCTION}

Thermal deburring, also known as Thermal Energy Method (TEM), is a process of removing burrs that are caused by different procedures (i.e. drilling, milling etc.) during the surface formation in machinery and related areas. It was ranked among the top of the most convenient and effective methods of deburring, due to a low performing time, simple automation, absence of abrasive inclusions after the processing and removing both internal and external located burrs [1? , 2].

The heat source varies according to instrument: edge finishing laser [3 5], electron beam melting (EBM) 6, electric and plasma arc [7, high frequency currents heating [8]; nevertheless, the main common drawback of these methods is still the necessity for a high accuracy of positioning to the processed detail, heating intensity and performance time of aforementioned heat sources.

All of these treatment methods show satisfactory results in external surface finishing, but in terms of internal defects processing treatment of processed part with combustion gases have numerous advantages in performance, accuracy and reliability [1, 9, 10. In addition, the TEM is used in an automatic mode using a CNC (computer numerical control) deburring machine. The main settings of this machine are a composition of the gas mixture, its initial pressure and exposure time. The specified parameters should provide the values of heat fluxes required for process- ing and their uniform interaction with the surfaces of the part.

Nowadays, the developing of operation settings of thermal treatment for the CNC deburring machine could be represented by two different approaches. The experimental path utilizes the data from previous experiments, but obtained empirical equations cannot provide accurate results and ensure the quality of the ultimate product. Another way is a full numerical simulation of the deburring process using combustion gases. In this case, a careful consideration of all particular processes (i.e. combustion of initial gas mixture, shock waves formation, and heat distribution) should be included, making this method excessively costly for complicated and precise parts.

In current work, we propose an alternative way for the thermal deburring process simulation based on a simplified model, with some corrections from empirical data. The model was built with the assumption of the possibility to replace a real chamber with a complex shape part on the chamber with an equivalent volume. The result of the theoretical and experimental study of heat fluxes, shock waves propagation in combustion chamber in the presence of processed part is represented below.

\section{MAThematiCAL MODEL}

The simulation of deburring by combustion gases was performed with transient analysis. 
Taking into account the complicated nature of the modelled system, some assumptions were used:

- A premixed flame is used.

- 2D axisymmetric case is considered.

- Reynolds decomposition is used to simplify NavierStokes equations.

A commercial code ANSYS Fluent was used for numerical simulations [12].

The geometry as well as the mesh was created via Workbench platform, a model was created according to the dimensions of the experimental tube. The mesh size was chosen according to the expected velocities magnitude. Several simulations were done to check the case sensitivity on a mesh size and to obtain a discretization error using a grid convergence index. It was found out that the difference in heat flux value between one and 10 million of elements is less than $1 \%$. Initially, a mesh with half a million of elements was used, so after the mesh dependence check, we found out that 1 million of cells is enough to obtain an accurate solution with a minimal computational time.

The combustion of gases is governed by a set of equations describing the conservation of mass, momentum, energy, and species. Excluding the mass forces, baro - and thermal diffusion, the equations are given by:

$$
\begin{gathered}
\frac{\partial \rho}{\partial t}+\nabla \cdot(\rho \cdot \mathbf{u})=0 \\
\frac{\partial(\rho \cdot u)}{\partial t}+\nabla \cdot(\rho \cdot u \cdot u)=-\nabla P+\nabla \cdot \tau_{e f f} \\
\frac{\partial(\rho h)}{\partial t}+\nabla \cdot(u \rho h)=\frac{\partial P}{\partial t}+u \cdot \nabla P+ \\
+\nabla\left(\lambda_{e f f} \nabla T-\sum_{i} h_{i} J_{i}+\tau_{e f f} \cdot u\right)+ \\
+\sum_{i=1}^{N} Q^{i}-Q_{r a d} \\
\frac{\partial\left(\rho Y_{i}\right)}{\partial t}+\nabla \cdot\left(\rho u Y_{i}\right)= \\
=\nabla \cdot\left(\left(\rho D_{i}+\frac{\mu_{t}}{S c_{t}}\right) \nabla Y_{i}\right)+R_{i}
\end{gathered}
$$

where $\rho$ is the density, $\left[\mathrm{kg} / \mathrm{m}^{3}\right] ; \mathbf{u}$ is the velocity vector; $t$ is the time, $[\mathrm{s}] ; P$ is the pressure, $[\mathrm{Pa}]$; $\tau_{\text {eff }}=\left(\mu+\mu_{t}\right)\left(\nabla u+(\nabla u)^{T}-2 / 3 I \cdot(\nabla \cdot u)\right)$ is the effective stress tensor (i.e., the sum of the viscous and turbulent stresses); $\mu$ is the viscosity, $[\mathrm{Pa} \cdot \mathrm{s}] ; \mu_{t}$ is the turbulent viscosity; $\mathbf{I}$ is the unit tensor; $h$ is the enthalpy of the mixture, $[\mathrm{J}] ; \lambda_{e f f}=\lambda+\lambda_{t}$ is the effective thermal conductivity; $\lambda$ is the laminar heat conductivity $\left[\mathrm{J} /(\mathrm{m} \cdot \mathrm{s} \cdot \mathrm{K}] ; \lambda_{t}=C_{p} \mu_{t} P r_{t}^{-1}\right.$ is the turbulent heat conductivity; $C_{p}$ is the specific heat, $[\mathrm{J} / \mathrm{K}]$;
$\mathrm{Pr}_{t}$ is turbulent Prandtl number; $T$ is the temperature, $[\mathrm{K}]$; , where $T_{\text {ref }}$ is $298.15 \mathrm{~K}$, is the enthalpy of species i, $[\mathrm{J}] ; \mathbf{J}_{i}$ is the diffusion rate vector of species i; $Q^{i}=h_{i}^{0} R_{i} / M_{i}$ is the heat of the chemical reactions that the species i participate in, $[\mathrm{J}] ; Q_{\text {rad }}$ is the radiation heat, $[\mathrm{J}] ; Y_{i}$ is the mass fraction of species $\mathrm{i} ; D_{i}$ is the diffusion coefficient of species $i$ in the mixture, $\left[\mathrm{m}^{2} / \mathrm{s}\right] ; S c_{t}$ is the turbulent Schmidt number; $R_{i}$ is the rate of the production of species $i$ by the chemical reaction.

For the current study, the SST turbulence model was used, which shows good results simulating the wall surface flows. According to this model, the turbulent viscosity is calculated as [11:

$$
\mu_{T}=\frac{0.31 \rho k}{\max \left(0.31 \omega ; \Omega F_{2}\right)}
$$

where $F_{2}=\tanh \left(\arg _{2}^{2}\right) ; \arg _{2}=\max \left(2 \frac{\sqrt{k}}{0.09 \omega y} ; \frac{500 \nu}{y^{2} \omega}\right)$ - function equals one for the boundary layer, and it equals zero for the free layer; $\Omega=(\partial u / \partial n)$ - is the derivative of the flow rate on the normal to the wall; $k$ and $\omega$ are the turbulence kinetic energy and the specific dissipation rate, respectively.

Parameters $k$ and $\omega$ were obtained from the following transport equations:

$$
\begin{aligned}
\frac{\partial \rho k}{\partial t}+\frac{\partial}{\partial x_{i}}\left(\rho v_{i} k\right)= & \tau_{i j} \frac{\partial v_{i}}{\partial x_{j}}-\beta^{*} \rho \omega k+ \\
& +\frac{\partial}{\partial x_{i}}\left(\left(\mu+\sigma_{k} \mu_{t}\right) \frac{\partial k}{\partial x_{i}}\right), \\
\frac{\partial \rho \omega}{\partial t}+\frac{\partial}{\partial x_{i}}\left(\rho v_{i} \omega\right) & =\frac{\gamma}{\nu_{\tau}} \tau_{i j} \frac{\partial v_{i}}{\partial x_{j}}-\beta \rho \omega^{2}+ \\
+\frac{\partial}{\partial x_{i}}( & \left.\left(\mu+\sigma_{\omega} \mu_{t}\right) \frac{\partial \omega}{\partial x_{i}}\right)+ \\
& +2 \rho\left(1-F_{1}\right) \sigma_{\omega 2} \frac{1}{\omega} \frac{\partial k}{\partial x_{j}} \frac{\partial \omega}{\partial x_{j}},
\end{aligned}
$$

where $\nu_{\tau}=\frac{a_{1} k}{\max \left(a_{1} \omega ; \partial u / \partial y F_{2}\right)} ;$ model constant $\phi_{S S T}$ $\left(\gamma, \sigma_{k}, \sigma_{\omega}, \beta, \beta^{*}\right)$ associated with $k-\omega$ model constants $\phi_{k \omega}$ and transformed $k-\epsilon$ model $\phi_{k \epsilon}$ as: $\Phi_{S S T}=\Phi_{k \omega} F_{1}+\left(1-F_{1}\right) \Phi_{k \varepsilon} ; F_{1}=\tanh \left(\arg _{1}^{4}\right) ;$ $\arg _{1}=\min \left[\max \left(\frac{\sqrt{k}}{0.09 \omega y} ; \frac{500 \nu}{y^{2} \omega}\right) ; \frac{4 \rho \sigma_{\omega 2} k}{C D_{k \omega} y^{2}}\right] ; y-$ distance to the nearest wall, $[\mathrm{m}] ; C D_{k \omega}=$ $\max \left(2 \rho \sigma_{\omega 2} \frac{1}{\omega} \frac{\partial k}{\partial x_{j}} \frac{\partial \omega}{\partial x_{j}}, 10^{-20}\right)$.

The net source of chemical species $i$, due to the fact that the reaction is computed as the sum of the Arrhenius reaction sources over the NR reactions that the species participate in:

$$
R_{i}=M_{i} \sum_{r=1}^{N_{R}} \hat{R}_{i, r}
$$

where $M_{i}$ is the molecular weight of species $i$, $[\mathrm{kg} / \mathrm{mol}]$ and $\hat{R}_{i, r}$ is the Arrhenius molar rate of creation/destruction of species $i$ in reaction $r,[\mathrm{~mol} / \mathrm{s}]$. 
For a reversible reaction, the molar rate of creation/destruction of species $i$ in the reaction $r$ is given by 12$]$ :

$$
\begin{aligned}
& \hat{R}_{i, r}=J\left(\nu_{i, r}^{\prime \prime}-\nu_{i, r}^{\prime}\right) \\
& \left(k_{f, r} \prod_{j=1}^{N}\left[C_{j, r}\right]^{\eta_{j, r}^{\prime}}-k_{b, r} \prod_{j=1}^{N}\left[C_{j, r}\right]^{\eta_{j, r}^{\prime \prime}}\right),
\end{aligned}
$$

where $N$ is the number of chemical species in the system; $\nu_{i, r}^{\prime}$ is the stoichiometric coefficient for the reactant $i$ in the reaction $r ; \nu_{i, r}^{\prime \prime}$ is the stoichiometric coefficient for the product $i$ in the reaction $r ; k_{f, r}$ is the forward rate constant for the reaction $r ; k_{b, r}$ is the backward rate constant for the reaction $r$; $J$ represents the net effect of third bodies on the reaction rate; $C_{i, r}$ is the molar concentration of each reactant and product species $j$ in reaction $r\left(\mathrm{~kg} \mathrm{~mol} / \mathrm{m}^{3}\right) ; \eta_{j, r}^{\prime}, \eta_{j, r}^{\prime \prime}$ forward and backward rate exponent for each reactant and product species $j$ in reaction $r$ respectively.

To determine the constants of direct and backward reactions, the Arrhenius equals have been used [13]:

$$
\begin{aligned}
& k_{f, r}=A_{r 1} T^{\beta_{r 1}} \exp \left(-\frac{E_{r}}{R T}\right), \\
& k_{b, r}=A_{r 2} T^{\beta_{r 2}} \exp \left(-\frac{E_{r}}{R T}\right),
\end{aligned}
$$

where $A_{r}, \beta_{r}$ - are the pre-exponential factor and the temperature exponent; $E_{r}$ is the activation energy for the reaction, $[\mathrm{J} / \mathrm{mol}] ; R$ is the universal gas constant, $[\mathrm{J} /(\mathrm{mol} \cdot \mathrm{K})]$.

In the presented paper, the combustion simulation was carried for the commonly used fuel gases: hydrogen, methane and propane, and oxidants: oxygen and air. Methane-air combustion mechanism consists of 17 species and 37 reactions, propane-air mechanism - 35 species and 108 reactions and hydrogen-air mechanism - 8 species and 40 reactions. For the convective heat flux calculation, the formulation of Kader has been applied [14, 15]:

$$
\begin{aligned}
T^{+}=\operatorname{Pr} & \cdot \tilde{y}^{+} \exp (-\Gamma)+ \\
& +\left[2.12 \ln \left(1+\tilde{y}^{+}\right)+\beta\right] \exp (-1 / \Gamma),
\end{aligned}
$$

where $\beta=\left(3.85 \operatorname{Pr}^{1 / 3}-1.3\right)^{2}+2.12 \ln (\operatorname{Pr}) ; \Gamma=$ $\frac{0.01\left(\operatorname{Pr} \cdot \tilde{y}^{+}\right)^{4}}{1+5 \operatorname{Pr} \cdot \tilde{y}^{+}}$.

In expression 12 dimensionless temperature was used:

$$
T^{+}=\frac{\rho c_{p} \tilde{u}_{\tau}\left(T_{w}-T_{f}\right)}{q_{w}},
$$

where $T_{w}$ - wall temperature, $[\mathrm{K}] ; T_{f}$ - temperature of combustion products in the flow core, $[\mathrm{K}] ; q_{w}$ - the wall heat flux, $\left[\mathrm{W} / \mathrm{m}^{2}\right] ; \tilde{u}_{\tau}=\left[\left(\tilde{u}_{\tau}^{v i s}\right)^{4}+\left(\tilde{u}_{\tau}^{\log }\right)^{4}\right]^{0.25}$ - the velocity profile in the boundary layer; $\tilde{u}_{\tau}^{v i s}=\frac{U_{1}}{\tilde{y}^{+}}$- the velocity in the viscous sublayer; $\tilde{u}_{\tau}^{\log }=\frac{U_{1}}{(1 / k) \ln \left(\tilde{y}^{+}\right)+C}-$ the velocity in logarithmic sublayer; $y^{+}$- dimensionless distance from the first grid node to the wall; $\tilde{y}^{+}=$ $\max \left(y^{+}, 11.067\right)$, the limiting value of $y^{+}=11.067$ marks the intersection between the logarithmic and the linear profile; $U_{1}$ - the flow rate in the node, which is the nearest to the wall.

The calculation grid near the wall was built according to the recommendations for $y^{+}$grid spacing for SST model. In order to cope with high gradients of pressure and velocity a time step of 1e-6s was used for the pressure based solver.

The value of the heat flux was calculated with use Eq. 13):

$$
q_{w}=\frac{\rho c_{p} \tilde{u}_{\tau}}{T^{+}}\left(T_{w}-T_{f}\right)
$$

The radiation is calculated by $\mathrm{P} 1$ radiation model [16.

\section{NUMERICAL SIMULATION AND EXPERIMENTAL ANALYSIS}

To evaluate the accuracy of the proposed model, a set of experiments was performed. An experimental investigation of deflagration-to-detonation transition in a tube with obstacles was carried out [17]. An experimental setup for the detonation study is shown in Figure 1. The main part of the installation is a circular cross-section detonation tube, made of stainless steel 1X18H9T. The general scheme of the installation is shown on Figure 2 The tube (1) has a total length $L=6 \mathrm{~m}$ and an internal diameter $D=0.14 \mathrm{~m}$ consists of four sections $(2,2,1$ and $1 \mathrm{~m})$ connected together. Thin ring-shape obstacles (2) with an axial hole diameter $d=0.108 \mathrm{~m}$ are installed inside the tube. The considered pipe locking ratio is equal to 0.4 .

The number of obstacles is 35 rings, which are arranged in increments equal to $S=0.14 \mathrm{~m}$ (equal to the diameter of the tube) and connected with each other by $0.015 \mathrm{~m}$ diameter rods to ensure the constancy of the interval. Figure 3 shows the scheme of the obstacles location.

To ensure the tightness of the detonation tube, its ends are closed with metal flanges. Diameter of the flanges is equal to $0.26 \mathrm{~m}$ and they are made from a material similar to the pipe.

PCB Piezotronics pressure sensors (10), which are located along the tube at a distance of $1.25,, 750 ; 2.75$; $3.25 ; 3.75 ; 4,625$ and $5,125 \mathrm{~m}$ from the entrance end, were used to monitor the pressure. The experimental results were obtained and processed using an automatic data collection system and a personal computer (3).

Before filling the tube, the fuel mixture was previously prepared in a cylinder (5), the air from the detonation tube was pumped out with a vacuum pump (6) to a vacuum at pressure $10 \mathrm{~Pa}$.

Ignition of the mixture was carried out using an automobile spark plug (8) and a time sequence generating device (4). The spark plug is connected to the ignition device (7) and is mounted on the flange, which closes the inlet end of the pipe. The release 

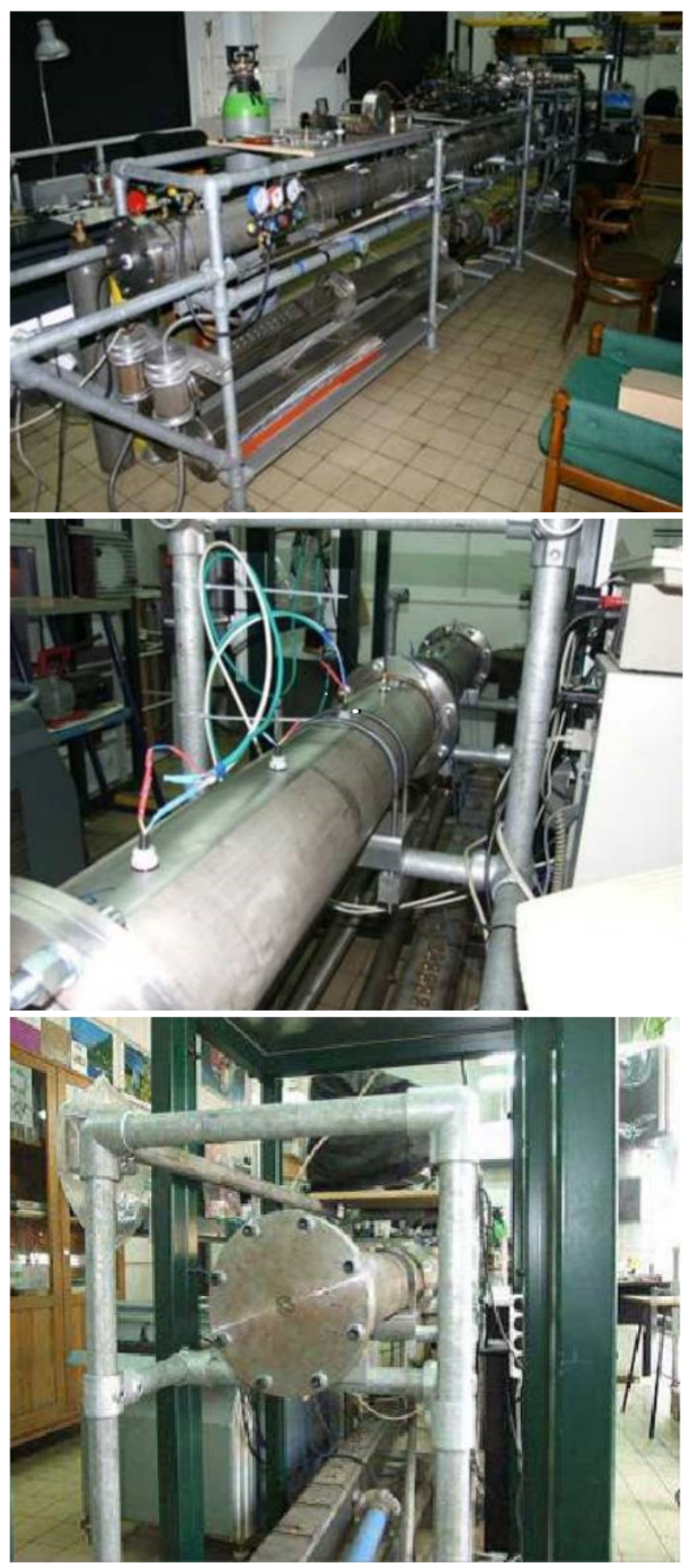

FigURE 1. Experimental setup for the detonation study.

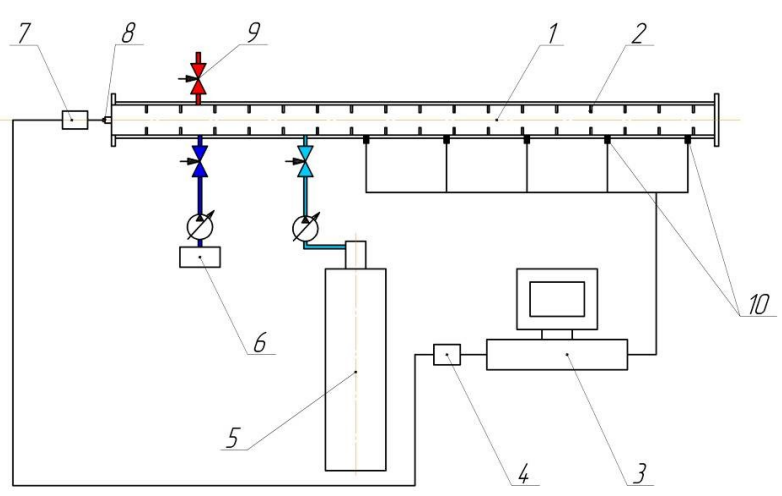

Figure 2. The scheme of the installation: 1 - detonation tube; 2 - obstacle; 3 - automatic data collection system and a personal computer; 4 - time sequence generating device ; 5 -cylinder filled with air-hydrogen mixture; 6 - vacuum pump; 7 - ignition device; 8 spark plug; 9 - exhaust valve; 10 - pressure censor.
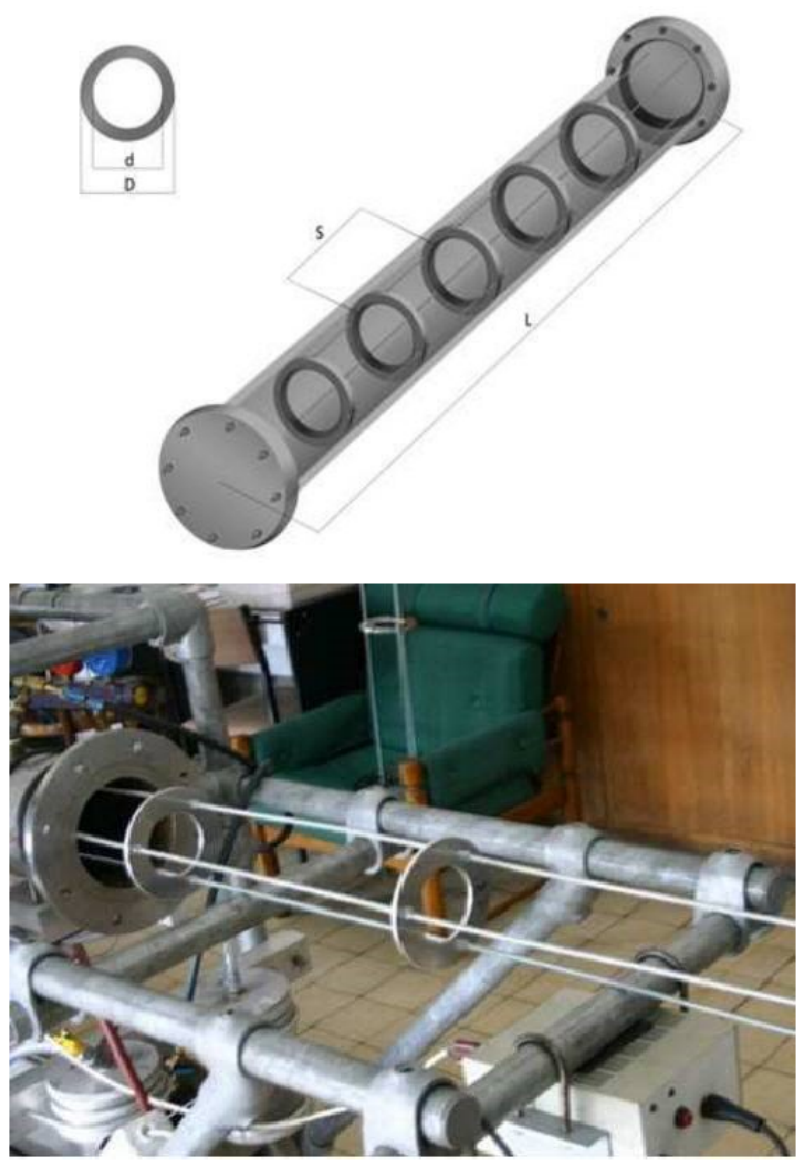

Figure 3. Scheme of the obstacles location. 


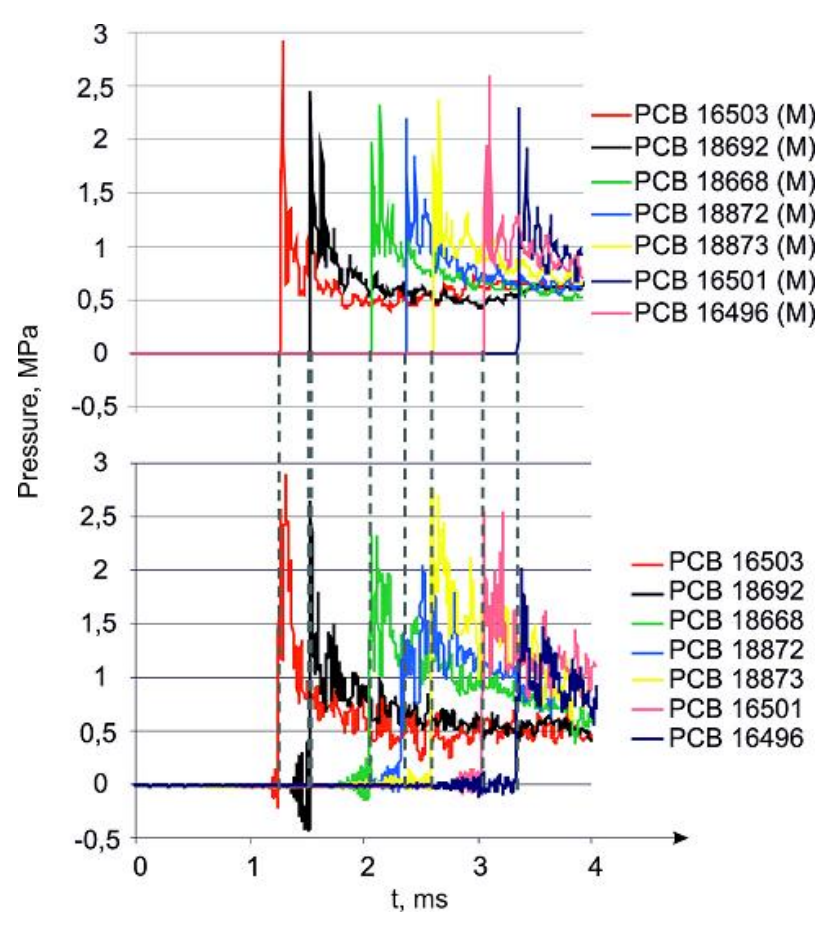

Figure 4. Calculated (top) and experimental (bottom) pressure profiles.

of combustion products was carried out through the exhaust valve $(9)$.

Obtained experimental and simulations results are shown in the Figure 4 and 5 , demonstrating the convergence of pressure and detonation velocity values in both cases (difference between pressure and time peak values do not exceed $3 \%$ and $5 \%$ respectively). The same accuracy results were obtained in measurements of shock waves damping and the in-chamber heat exchange for the acetylene-oxygen and methane-oxygen mixtures combustion, showing the applicability of the proposed model in further investigations.

Further work was carried out to study the thermal deburring of parts with complex shapes by combustible gas mixtures. As the main hypothesis, it was assumed that, to determine the required heat flux and damping time of the shock waves in the chamber for treatment parts with a complex shape, it is possible to use calculation results from the simplified model with an equivalent chamber.

As an equivalent chamber, two cases have been considered. The first case is an empty chamber, the volume of which is defined as $V_{e q v_{c} h a m}=V_{\text {cham }}-$ $V_{\text {parts }}$ (Figure 6a), where $V_{\text {eqv }}$ ham $, V_{\text {cham }}, V_{\text {parts }}$ the volume of the equivalent chamber, the volume of the real chamber and the volume of treatment parts, respectively. The second case is the chamber with simple geometric shaped parts located inside, for

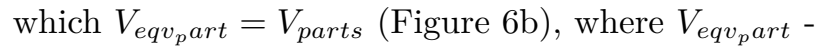
the volume of the equivalent part.

At least three different modes can be distinguished for the combustion process inside a closed chamber - deflagration, detonative and knocking combustion. The heat transfer between the processed part and heat fluxes significantly differs for all cases, requiring a careful consideration of process conditions for a uniform distribution of heat along the part.

The lowest values of heat fluxes are achieved for deflagration. The main drawback of this approach is a high heterogeneity of temperature inside the chamber - the difference can reach more than $500 \mathrm{~K}$; this phenomenon is known as the Mache effect. The solution of this problem lies in a modification of the ignition source( i.e. corona discharge [18] or laser radiation [19]). In our previous work we describe the pre-chamber torch ignition, which results in homogeneous temperature in chamber with a standard deviation that does not exceed $1 \%$ [20].

The dependence of the heat fluxes on pressure can be estimated from the following considerations. The heat transfer coefficient in the thermal boundary layer can be described:

$$
\begin{aligned}
\alpha\left(T_{\text {mixture }}-T_{\text {wall }}\right)=\lambda \frac{\partial T}{\partial n} & \approx \\
& \approx \lambda \frac{\left(T_{\text {mixture }}-T_{\text {wall }}\right)}{\delta},
\end{aligned}
$$

where $\delta$ - the thickness of the thermal boundary layer, which is inversely proportional to the square root of the Reynolds number for laminar flow $\delta \sim 1 / \sqrt{\operatorname{Re}}$.

Assuming that the viscosity, temperature and velocity of the combustion products are weakly dependent on the initial state of the mixture: $\delta \sim 1 / \sqrt{\rho}$, and considering that $\rho=p / R T$, finally we have $\alpha \sim \sqrt{p / T}$.

For arbitrary initial pressure and temperature of the mixture, the equation for the heat flux determination will be:

$$
q_{p}=q_{p_{0}} \sqrt{p T_{0 \text { mixture }} / p_{0} T_{\text {mixture }}}
$$

where $-q_{p 0}$ heat flux, defined for the mixture with the initial pressure $p_{0}$ and temperature $T_{0}$.

The cyclic mode of a deburring machine work imposes more restrictions on the proposed model - periodic contact of combustion gases with chamber walls causes a rise of their temperature; additional heating of starting gas mixture affects the internal pressure, resulting in a fuel dosage error and an incorrect work of the deburring machine. To counterbalance this effect in our model, the simulations of additional heating impact on gas mixture dosage were performed using the mathematical model presented above, excluding the equations that describe chemical reactions. It was found out that temperature increase from $293 \mathrm{~K}$ to $423 \mathrm{~K}$ reduces the mass of the fuel by $12 \%$ at stable pressure conditions. It should be noted that mass control of the introduced fuel cannot be the solution of the aforementioned problem due to unknown starting temperature of the fuel mixture, and, as a result unknown final temperature. The most accurate way of the fuel dosage is a combined mass and pressure control. Using the latter values, it is possible to clearly determine the density and temperature of the starting 


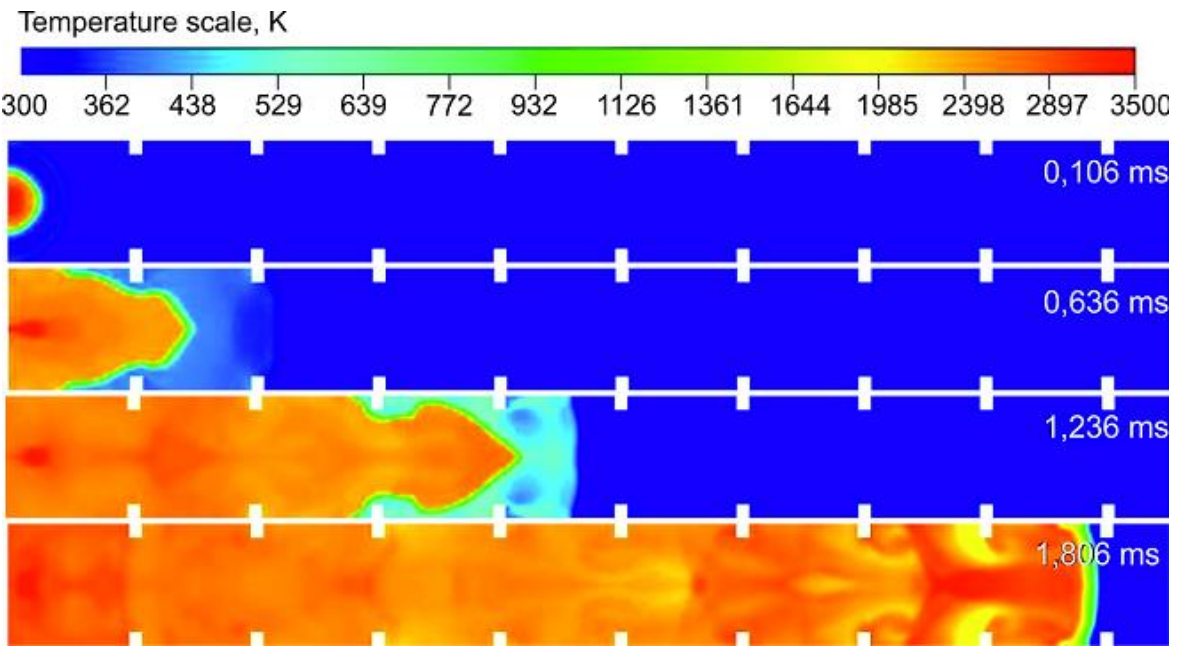

FiguRE 5. Simulation of flame propagation and deflagration-to-detonation transition in the pipe with obstacles.

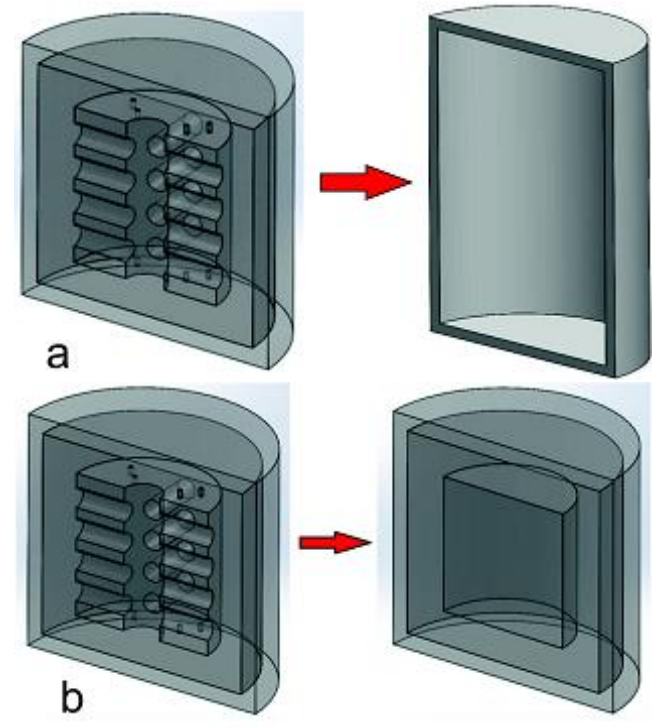

FigURE 6. Variants of the transition to an equivalent chamber.

gas mixture:

$$
\begin{gathered}
\rho_{\text {mix }}=m_{\text {mix }} /\left(V_{\text {cham }}-\text { Vparts }\right) \\
T_{\text {mix }}=p_{\text {mix }} / \Re_{\text {mix }} \rho_{\text {mix }},
\end{gathered}
$$

where $\Re_{m i x}=\sum_{i=1}^{N} Y_{i} \Re_{i}$, and $\Re_{i}$ - gas constant of the mixture components, $[\mathrm{J} /(\mathrm{kg} \cdot \mathrm{K})]$.

Considering the aforementioned fact, the time interval of the deburring process in a cyclic work mode can be described by following equations:

$$
t=t_{0} \frac{T_{a d}\left(T_{0}\right)}{T_{a d}\left(T_{m i x}\right)},
$$

where $t_{0}$ - processing time at flux $q_{p_{0}} ; T_{a d}$ - adiabatic combustion temperature at a constant volume corresponding to the initial temperature of the mixture.

Simulating the heat fluxes in the case of complex parts processing is a non-trivial problem; in this case,

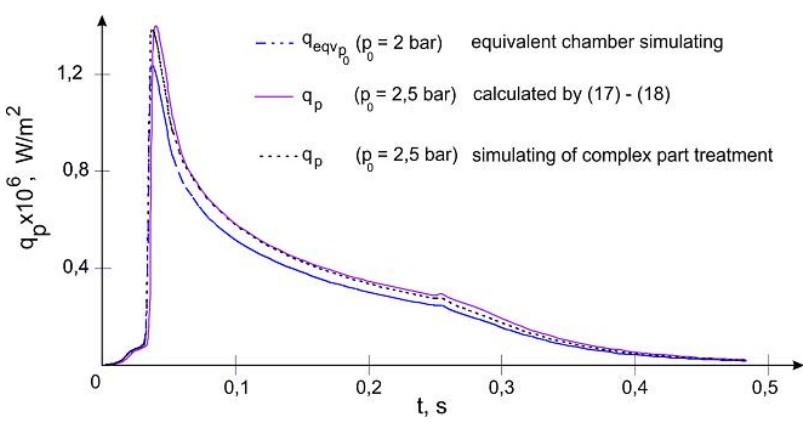

Figure 7 . Heat flux dependence on time during the surrounded combustion of the fuel mixture.

the conception of an equivalent chamber is an irreplaceable assistant in the calculation of heat flux $q_{p_{0}}$ instead of equation (15). The use of this model requires an amendment on the heat transfer surface area decrease; in assumption of a stable chamber walls temperature $(293 \mathrm{~K})$ the following equations for are presented (for models on Figure 6a and $6 \mathrm{p}$ respectively):

$$
\begin{gathered}
q_{p}=q_{\text {eqvp } 0} \sqrt{p T_{0} / p_{0} T_{p}} \frac{S_{\text {eqv_cham }}}{S_{\text {cham }}+S_{\text {parts }}}, \\
q_{p}=q_{\text {eqvp }_{0}} \sqrt{p T_{0} / p_{0} T_{p}} \frac{S_{\text {cham }}+S_{\text {eqv_part }}}{S_{\text {cham }}+S_{\text {parts }}} .
\end{gathered}
$$

Using relations $17-18$, the average heat flux through the part surface was determined for two general modes of the processing. First one is the case of a surrounded mixture combustion with a suppression of shock waves. Second one is the case of a mixture combustion with a shock waves formation and its damping after knocking combustion in the chamber. A numerical simulation for the combustion with the suppression of shock waves has been carried out for different initial pressures and different parts.

It should be noted that calculations of the heat flux using relationships (17) and (18) showed equal results with a deviation from experimental values being smaller than 2\%. Charts given in Figure 7 are typical for this series of experiments. 


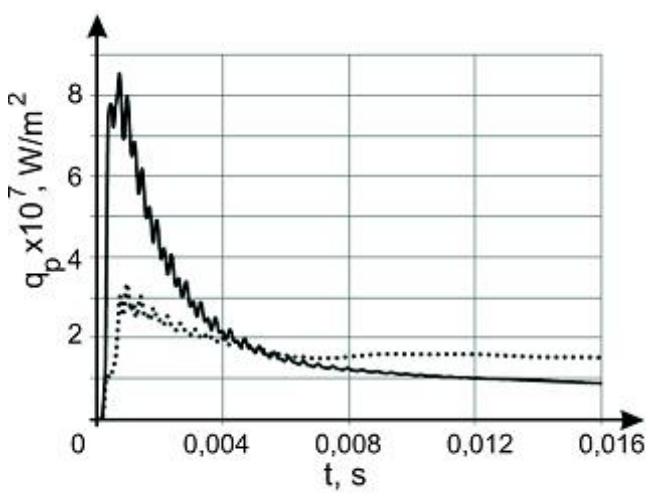

a

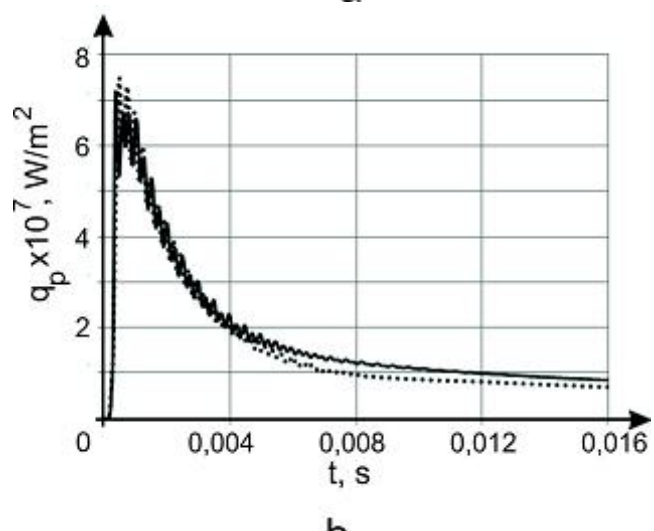

b

Figure 8. Heat flux dependence on time obtained for mixture combustion with shock waves formation.

The heat transfer in the combustion with the shock wave formation was performed previously [21]; in this case, the average heat transfer depends on the pressure by the following manner:

$$
q \sim \sqrt{p}
$$

Comparison of results for the direct numerical simulation and calculations with equations (17) and 18 shows a high significance (up to $5 \%$ ) of shock waves in the heat fluxes distribution (Figure $8 \mathrm{a}$ and $8 \mathrm{p}$ ).

The reflection of shock waves influences the magnitude and distribution of heat fluxes. To confirm this information, additional calculations for two-dimensional part were performed. Unsymmetrical positioning in chamber and variation of detonation place were used to differentiate the experimental data, obtained for different part surfaces. As shown in Figure 9 the uniform formation of shock waves along the vertical axis results in high differentiation of heat fluxes through non-equal edges with a perceptible periodicity of the impact time; whereas more chaotically formed shockwaves equalizes the heat flux on all edges. It should be noted that the combustion with shock waves formation increase the heat fluxes 10-100 times when compared to the deflagrative combustion, but it still needs a careful experimentation in order to provide the uniform processing mode.

The obtained results of the averaged heat flux and temperature of combustion products by the equivalent
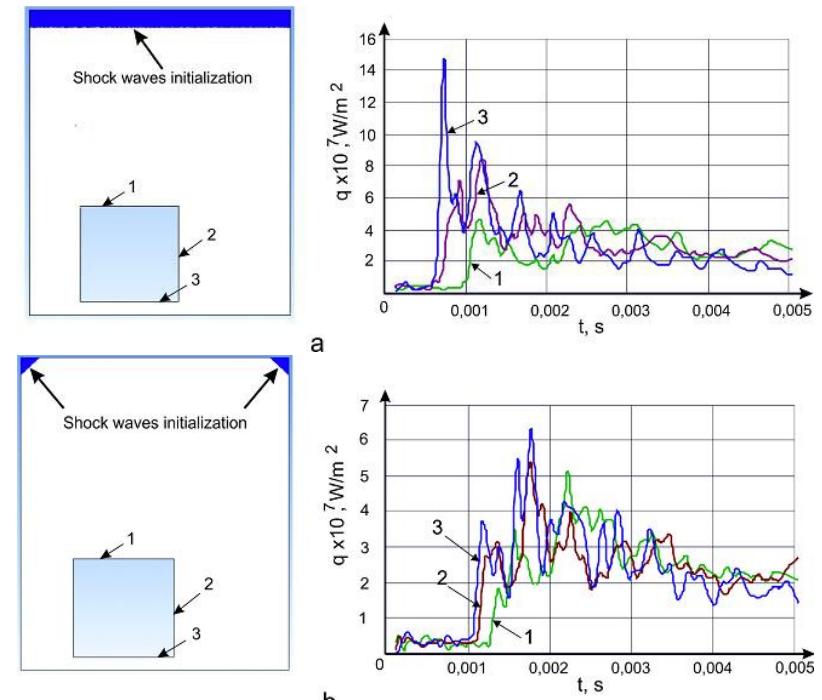

Figure 9. The influence of detonation mode on heat flux distribution on part surfaces.

chamber method allow us to evaluate an averaged heat transfer coefficient:

$$
\alpha=\frac{q_{e q v p_{0}}}{T_{e q v f}-293},
$$

where $T_{\text {eqv } f}$ - averaged temperature of the combustion products in an equivalent chamber.

Then, the heat flux for the arbitrary starting gas mixture pressure can be calculated by the following way:

$$
\begin{aligned}
q_{p}=\psi & \frac{q_{\text {eqvp }}}{T_{\text {eqvf }}-293} \sqrt{p T_{0} / p_{0} T_{p}} \\
& \frac{S_{\text {cham }}+S_{\text {eqv_part }}}{S_{\text {cham }}+S_{\text {parts }}}\left(T_{\text {eqv } f}-T_{\text {wall }}\right),
\end{aligned}
$$

where $T_{\text {wall }}$ - the part surfaces temperature changed during heating; $\psi$ - empirical coefficient determined experimentally, which includes the approximate nature of the proposed method of the equivalent chamber due to the absence of reliable data on the thermal characteristics of materials at temperatures near to the melting point. Our experience suggests that the definition of this coefficient requires a minimum number of experiments.

\section{Conclusions}

(1.) To determine the amount of the heat fluxes, which predicts the initial pressure of the gas mixture in the chamber and the exposure time for the thermal deburring process, the method with the use of the equivalent chamber conception was proposed. This approach does not exclude the necessity for experiments, however, it significantly reduces their quantity. Experimental and numerical simulations results were compared and it was found out that the maximum difference between the values was not more than $5 \%$. 
(2.) At first, an approximation of the arbitrary initial mixture pressure for the averaged heat flux on the part surfaces can be determined by the calculation results using obtained dependencies (17) or (18).

(3.) It was shown that different approaches towards the representation of the processed part contribution in heat fluxes propagation has a significant impact on the result of the theoretical study; the applicability of each model was demonstrated for the deflagrative combustion and the combustion with shock waves formation.

(4.) The most effective deburring effect was demonstrated by the combustion with chaotic shock waves distribution; nevertheless, this method requires some improvements to be introduced in practical use.

\section{REFERENCES}

[1] L. Gillespie. Deburring and Edge Finishing Handbook. Industrial Press, New York, 1999.

[2] J. Halladay. Practical applications of thermal deburring and electrochemical deburring. https://www.productionmachining.com/cdn/cms/ uploadedFiles/Practical $\% 20$ Applications $\% 20$ of $\%$ 20TD\%20and\%20ECD\%202011.pdf Accessed: 30 April 2019 .

[3] S. Lee. Analysis of precision deburring using a laser An experimental study and FEM simulation. KSME International Journal 14:141-151, 2000. DOI:10.1007/BF03184780.

[4] Y. Zhou, Y. Gao, B. Wu, et al. Deburring effect of plasma produced by nanosecond laser ablation. Journal of Manufacturing Science and Engineering 136:024501, 2014. DOI:10.1115/1.4025910.

[5] S. Lee, D. A. Dornfeld. Precision laser deburring. Journal of Manufacturing Science and Engineering 123:601-608, 1999. DOI:10.1115/1.1381007.

[6] H. Garich, T. D. Hall. Electrochemical surface finishing of additively manufactured parts. ECS Transactions 85(4):155-166, 2018. DOI:10.1149/08504.0155ecst

[7] R. I Valiev, A. A Khafizov, Y. I Shakirov, A. N Sushchikova. Polishing and deburring of machine parts in plasma of glow discharge between solid and liquid electrodes. IOP Conference Series: Materials Science and Engineering 86, 2015. DOI:10.1088/1757-899X/86/1/012026

[8] V. Koshuro, M. Fomina, A. Voyko, et al. Surface morphology of zirconium after treatment with high-frequency currents. Composite Structures 202, 2018. DOI:10.1016/j.compstruct.2018.01.055
[9] ASM International, and American Society for Metals. Metals handbook. Volume 16 - Machining. Metals Park, Ohio: ASM International., 1989.

[10] A. Fritz, L. Sekol, J. Koroskenyi, et al. Experimental analysis of thermal energy deburring process by design of experiment. In ASME 2012 International Mechanical Engineering Congress and Exposition. 2012. DOI:10.1115/IMECE2012-88411.

[11] F. Menter. Two-equation eddy-viscosity transport turbulence model for engineering applications. AIAA Journal 32:1598-1605, 1994. DOI:10.2514/3.12149

[12] J. Warnatz, U. Maas, R. Dibble. Combustion Physical and Chemical Fundamentals, Modeling and Simulation, Experiments, Pollutant Formation. Springer-Verlag, 2006.

[13] M. Ó Conaire, H. J. Curran, J. M. Simmie, et al. A comprehensive modeling study of hydrogen oxidation. International Journal of Chemical Kinetics 36(11):603-622, 2004. DOI:10.1002/kin.20036

[14] S. Yungster, M. J. Rabinowitz. Computation of shock-induced combustion using a detailed methane-air mechanism. Journal of Propulsion and Power 10:609-617, 1994. DOI: $10.2514 / 3.23770$

[15] B. Kader. Temperature and concentration profiles in fully turbulent boundary layers. International Journal of Heat and Mass Transfer 24(9):1541-1544, 1981. DOI:https://doi.org/10.1016/0017-9310(81)90220-9

[16] M. Modest. Radiative Heat Transfer. Academic Press, 2013.

[17] W. Rudy, R. Porowski, A. Teodorczyk. Propagation of hydrogen-air detonation in tube with obstacles. Journal of Power Engineering 91:122-129, 2011.

[18] J. B. Miet, J. Lykowski, P. K. Mixell. Corona ignition system for highly efficient gasoline engines. $M T Z$ worldwide 74, 2013. DOI:10.1007/s38313-013-0062-z

[19] M. Weinrotter, H. Kopecek, E. Wintner, et al. Application of laser ignition to hydrogen-air mixtures at high pressures. International Journal of Hydrogen Energy 30:319-326, 2005. DOI:10.1016/j.ijhydene.2004.03.040

[20] V. Shypul, Y. Tsegelnik, V. Malashenko, S. Krasovskyy. Methodology for the selection parameters of pre-chamber for thermal-impulse treatment of plastic parts. Open informational and computer technologies 61:39-46, 2013.

[21] V. Manzhaley. The mechanics of reacting media and its applications, chap. Attenuation of shock waves and heat exchanger bodies after detonation in the gas chamber. Science, Novosibirsk, 1989. 